\title{
Axillary Giant Lipoma Excision under Serratus Plane Block
}

Ahmet Murat Yayik Ali Ahiskalioglu 2 Erkan Cem Celik 1 Sinan Celik 1 Akın Inaloz 3

1 Anesthesiology and Reanimation, Regiona Training and Research Hospital, Erzurum, Turkey

2 Anesthesiology and Reanimation, Ataturk University, Erzurum, Turkey 3 Plastic Surgery, Regional Training anc Research Hospital, Erzurum, Turke

\section{ESRA \\ DUBLIN, IRELAND}

EONTAGT

Ahmet Murat Yayik Eaning and Research Hospital, Email: m_yayik@ hotmail.com
Phone: 905544259287
Background and Aims

Serratus plane block(SPB) achieves complete analgesia of the hemithorax and axillary region. This block was generally used for acute postoperative pain management and reducing postoperative opioid consumption, neuropathic and chronic pain, however the use of surgical anesthesia is limited.

We report successful case of giant lipoma excision on axillary region with SPB for perioperative anesthesia and analgesia. A written consent form was obtained from the patients.
Methods

Case was an 18 year-old man, who under-went giant lipoma excision $(23 \mathrm{~cm} \times 10 \mathrm{~cm} \times 5 \mathrm{~cm})$ on theaxillary region (Fig. 1A and B).

The latissimus dorsi and serratus muscles were visualized at the level of 4th and5th ribs on the posterior-axillary line. With the in-planetechnique, a $100 \mathrm{~mm}$ sonovisible nerve block needle wasadvanced between the latissimus dorsi and the serratus mus-cles planes in a caudal to the cranial direction.

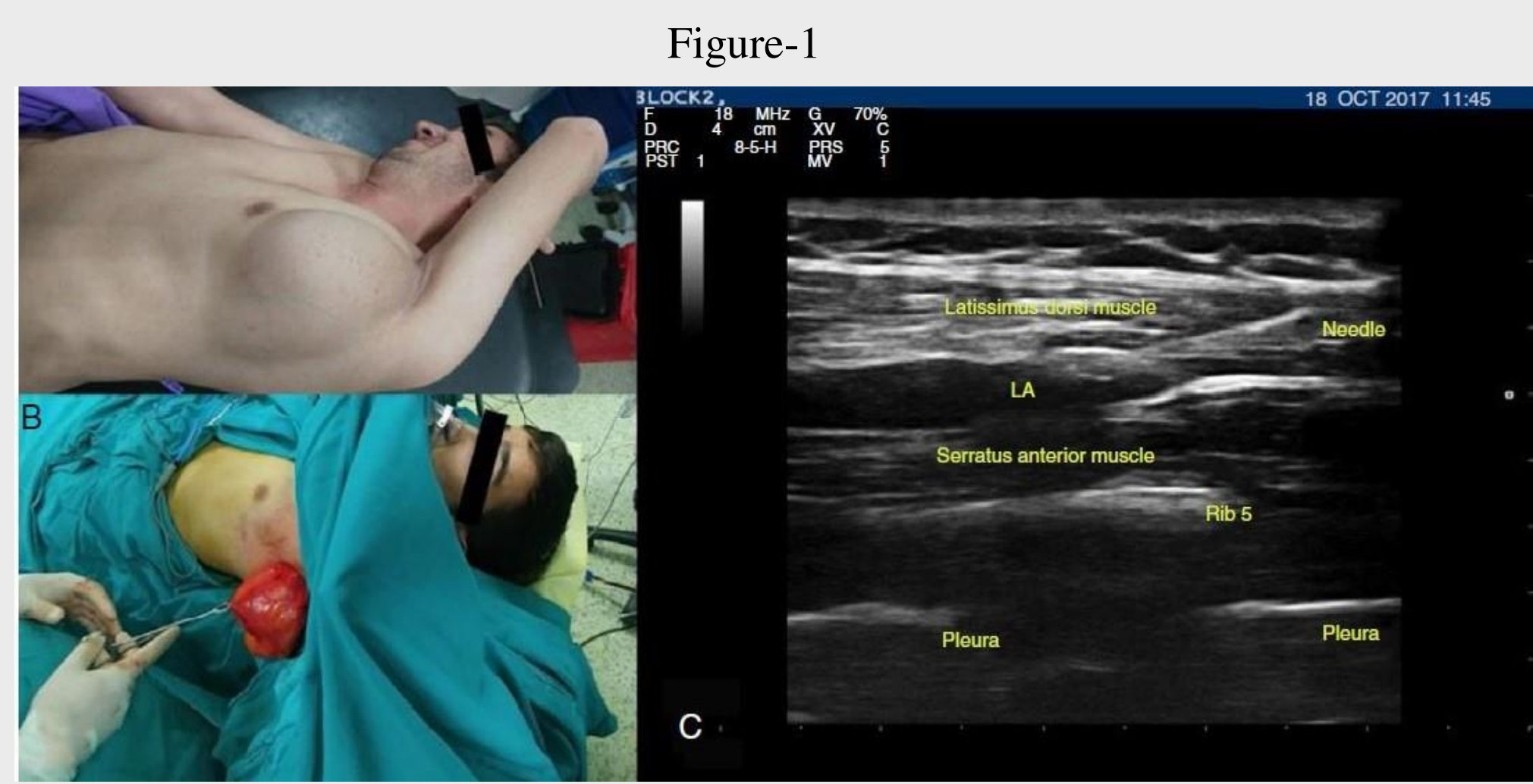

Two $\mathrm{mL}$ saline injection was made to confirm the position of the needle and $15 \mathrm{~mL} 0.5 \%$ bupivacaine and $15 \mathrm{~mL} 2 \%$ lidocaine injection between the two muscles (Fig. 1C)

Anesthesia was provided to the thoracic wall from Th2 to Th8 and axillary region. Surgery was uneventful and no additional medication was needed for intraoperative period.

\section{Conclusions}

Many nerves participate in the innervation of the axillary region (intercostobrachialis, intercostal, long thoracic, andthoracodorsal nerves). The intercostobrachialis nerve is major nerve of axilla and SPB is surely effective for this nerve. In our clinic, we have performed SPB successfully for exci-sional axillary lymph node biopsies for surgical anesthesia at our unpublished experiences.

SPB may be an alternative to general anesthesia, especially in axillary and thoracic wall superficial lesions.

REFERENGES

Torre PA, Jones JW Jr, Alvarez SL, et al Axillary localanesthetic spread
after the thoracici interfacial lutrasoundollock-..- a cadaveric and radiological

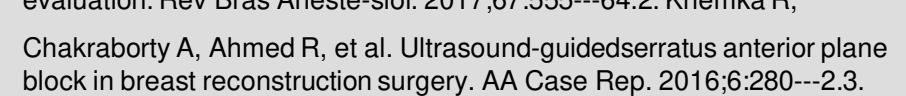

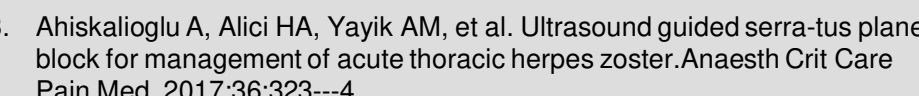

